\title{
Stability of Impulsive Neural Networks with Time-Varying and Distributed Delays
}

\author{
Qi Luo, ${ }^{1}$ Xinjie Miao, ${ }^{1}$ Qian Wei, ${ }^{1}$ and Zhengxin $Z_{h o u}{ }^{2}$ \\ ${ }^{1}$ School of Information \& Control, Nanjing University of Information Science \& Technology, Nanjing, Jiangsu 210044, China \\ ${ }^{2}$ School of Mathematical Science, Yangzhou University, Yangzhou, Jiangsu 225009, China \\ Correspondence should be addressed to Qi Luo; hb2-102@nuist.edu.cn
}

Received 26 December 2012; Accepted 21 March 2013

Academic Editor: Xuerong Mao

Copyright (c) 2013 Qi Luo et al. This is an open access article distributed under the Creative Commons Attribution License, which permits unrestricted use, distribution, and reproduction in any medium, provided the original work is properly cited.

\begin{abstract}
This work is devoted to investigating the stability of impulsive cellular neural networks with time-varying and distributed delays. We use the new method of fixed point theory to obtain some new and concise sufficient conditions to ensure the existence and uniqueness of solution and the global exponential stability of trivial equilibrium. The presented algebraic criteria are easily checked and do not require the differentiability of delays.
\end{abstract}

\section{Introduction}

Since cellular neural networks (CNNs) were proposed by Chua and Yang in 1988 [1, 2], many researchers have put great effort into this subject due to their numerous successful applications in various fields such as optimization, linear and nonlinear programming, associative memory, pattern recognition, and computer vision.

Owing to the finite switching speed of amplifiers, there is no doubt that time delays exist in the communication and response of neurons. Moreover, as neural networks usually have a spatial extent due to the presences of a multitude of parallel pathways with a variety of axon sizes and lengths, there is a distribution of conduction velocities along these pathways and a distribution of propagation designed with discrete delays. Therefore, a more appropriate and ideal way is to incorporate continuously distributed delays with a result that a more effective model of cellular neural networks with time-varying and distributed delays proposed.

In fact, beside delay effects, stochastic and impulsive as well as diffusing effects are also likely to exist in neural networks. So far, there have been many results [3-11] on the study of dynamic behaviors of complex CNNs such as impulsive delayed reaction-diffusion CNNs and stochastic delayed reaction-diffusion CNNs. Summing up the existing researches on the stability of complex CNNs, we see that the primary method is Lyapunov theory. However, there are also lots of difficulties in the applications of corresponding theories to specific problems. It is therefore necessary to seek some new methods to deal with the stability in order to overcome those difficulties.

Recently, it is inspiring that Burton and other authors have applied the fixed point theory to investigate the stability of deterministic systems and obtained some more applicable conclusions, for example, see the monograph [12] and the work in [13-24]. In addition, more recently, there have been a few papers where the fixed point theory is employed to investigate the stability of stochastic (delayed) differential equations, for instance, see [25-31]. Precisely, in [26-28], Luo used the fixed point theory to study the exponential stability of mild solutions for stochastic partial differential equations with bounded delays and with infinite delays. In $[29,30]$, Sakthivel used the fixed point theory to discuss the asymptotic stability in pth moment of mild solutions to nonlinear impulsive stochastic partial differential equations with bounded delays and with infinite delays. In [31], Luo used the fixed point theory to study the exponential stability of stochastic Volterra-Levin equations. We wonder if we can obtain some new and more applicable stability criteria of complex CNNs by applying the fixed point theory.

With this motivation, in this paper, we aim to discuss the global exponential stability of impulsive CNNs with timevarying and distributed delays. It is worth noting that our research technique is based on the contraction mapping 
principle rather than the usual method of Lyapunov theory. We deal with, by employing the fixed point theorem, the existence and uniqueness of solution and the global exponential stability of trivial equilibrium at the same time, for which Lyapunov method feels helpless. The obtained stability criteria are easily checked and do not require the differentiability of delays.

\section{Preliminaries}

Let $R^{n}$ denote the $n$-dimensional Euclidean space and $\|\cdot\|$ represent the Euclidean norm $\mathcal{N} \triangleq\{1,2, \ldots, n\}$ and $R_{+}=$ $[0, \infty) . C[X, Y]$ corresponds to the space of continuous mappings from the topological space $X$ to the topological space $Y$.

In this paper, we consider the following impulsive cellular neural networks with time-varying and distributed delays:

$$
\begin{aligned}
& \frac{\mathrm{d} x_{i}(t)}{\mathrm{d} t}=-a_{i} x_{i}(t)+\sum_{j=1}^{n} b_{i j} f_{j}\left(x_{j}(t)\right) \\
&+\sum_{j=1}^{n} c_{i j} g_{j}\left(x_{j}\left(t-\tau_{i j}(t)\right)\right) \\
&+\sum_{j=1}^{n} d_{i j} \int_{0}^{\rho(t)} \sigma_{j}\left(x_{j}(t-\theta)\right) \mathrm{d} \theta \\
& t \geq 0, t \neq t_{k}, \\
& \Delta x_{i}\left(t_{k}\right)= x_{i}\left(t_{k}+0\right)-x_{i}\left(t_{k}\right)=I_{i k}\left(x_{i}\left(t_{k}\right)\right), \\
& k=1,2, \ldots,
\end{aligned}
$$

where $i \in \mathscr{N}$ and $n$ is the number of neurons in the neural network. $x_{i}(t)$ corresponds to the state of the $i$ th neuron at time $t . f_{j}, g_{j}$, and $\sigma_{j}$ denote the activation functions, respectively. The constant $a_{i}>0$ represents the rate with which the $i$ th neuron will reset its potential to the resting state when disconnected from the network and external inputs. The constants $b_{i j}, c_{i j}$, and $d_{i j}$ represent the connection weights of the $j$ th neuron to the $i$ th neuron, respectively. $\tau_{i j}(t)$ and $\rho(t)$ correspond to the transmission delays meeting $0 \leq \tau_{i j}(t) \leq \tau$ ( $\tau=$ constant $)$ and $0 \leq \rho(t) \leq \rho$ ( $\rho=$ constant $)$. The fixed impulsive moments $t_{k}(k=1,2, \ldots)$ satisfy $0=t_{0}<t_{1}<$ $t_{2}<\cdots$ and $\lim _{k \rightarrow \infty} t_{k}=\infty \cdot x_{i}\left(t_{k}+0\right)$ and $x_{i}\left(t_{k}-0\right)$ stand for the right-hand and left-hand limits of $x_{i}(t)$ at time $t_{k}$, respectively. $I_{i k}\left(x_{i}\left(t_{k}\right)\right)$ shows the impulsive perturbation of the $i$ th neuron at the impulsive moment $t_{k}$.

Throughout this paper, we always assume that $f_{i}(0)=$ $g_{i}(0)=\sigma_{i}(0)=I_{i k}(0)=0$ for $i \in \mathcal{N}$ and $k=1,2, \ldots$. Thereby, problems (1) and (2) admit a trivial equilibrium $\mathbf{x}=0$.

Denote by $\mathbf{x}(t) \triangleq \mathbf{x}(t ; s, \varphi) \quad=$ $\left(x_{1}\left(t ; s, \varphi_{1}\right), \ldots, x_{n}\left(t ; s, \varphi_{n}\right)\right)^{T} \in R^{n}$ the solution to (1) and (2) with the initial condition

$$
x_{i}(s)=\varphi_{i}(s), \quad-m^{*} \leq s \leq 0, i \in \mathcal{N},
$$

where $m^{*}=\max \{\tau, \rho\}, \varphi_{i}(s) \in C\left[\left[-m^{*}, 0\right], R\right]$ and $\varphi(s)=$ $\left(\varphi_{1}(s), \ldots, \varphi_{n}(s)\right)^{T} \in R^{n}$.

The solution $\mathbf{x}(t) \triangleq \mathbf{x}(t ; s, \varphi) \in R^{n}$ to (1)-(3) is, for the time variable $t$, a piecewise continuous vector-valued function with the first-kind discontinuous points $t_{k}(k=$ $1,2, \ldots)$, where it is left-continuous; that is, the following relations are true:

$$
\begin{array}{r}
x_{i}\left(t_{k}-0\right)=x_{i}\left(t_{k}\right), x_{i}\left(t_{k}+0\right)=x_{i}\left(t_{k}\right)+I_{i k}\left(x_{i}\left(t_{k}\right)\right), \\
\\
i \in \mathcal{N}, k=1,2, \ldots
\end{array}
$$

Definition 1. The trivial equilibrium $\mathbf{x}=0$ is said to be globally exponentially stable if for any initial condition $\varphi(s) \epsilon$ $C\left[\left[-m^{*}, 0\right], R^{n}\right]$, there exists a pair of positive constants $\lambda$ and $M$ such that

$$
\|\mathbf{x}(t ; s, \varphi)\| \leq M \mathrm{e}^{-\lambda t}, \quad \forall t \geq 0 .
$$

The consideration of this paper is based on the following fixed point theorem.

Theorem 2 (see [32]). Let $\Upsilon$ be a contraction operator on a complete metric space $\Theta$, then there exists a unique point $\zeta \in \Theta$ for which $\Upsilon(\zeta)=\zeta$.

\section{Main Results}

In this section, we will, for (1)-(3), use the contraction mapping principle to prove the existence and uniqueness of the solution and the global exponential stability of trivial equilibrium all at once. Before proceeding, we firstly introduce some assumptions as follows.

(A1) There exist nonnegative constants $l_{j}$ such that for any $\eta, v \in R$

$$
\left|f_{j}(\eta)-f_{j}(v)\right| \leq l_{j}|\eta-v|, \quad j \in \mathcal{N} .
$$

(A2) There exist nonnegative constants $k_{j}$ such that for any $\eta, v \in R$,

$$
\left|g_{j}(\eta)-g_{j}(v)\right| \leq k_{j}|\eta-v|, \quad j \in \mathcal{N} .
$$

(A3) There exist nonnegative constants $p_{j k}$ such that for any $\eta, v \in R$,

$$
\left|I_{j k}(\eta)-I_{j k}(v)\right| \leq p_{j k}|\eta-v|, \quad j \in \mathcal{N}, k=1,2, \ldots .
$$

(A4) There exist nonnegative constants $\omega_{j}$ such that for any $\eta, v \in R$,

$$
\left|\sigma_{j}(\eta)-\sigma_{j}(v)\right| \leq \omega_{j}|\eta-v|, \quad j \in \mathcal{N} .
$$

Let $\mathscr{H}=\mathscr{H}_{1} \times \cdots \times \mathscr{H}_{n}$, and let $\mathscr{H}_{i}(i \in \mathcal{N})$ be the space embracing functions $\phi_{i}(t):\left[-m^{*},+\infty\right) \rightarrow R$, wherein $\phi_{i}(t)$ satisfies the following:

(1) $\phi_{i}(t)$ is continuous on $t \neq t_{k}(k=1,2, \ldots)$,

(2) $\lim _{t \rightarrow t_{k}^{-}} \phi_{i}(t)$ and $\lim _{t \rightarrow t_{k}^{+}} \phi_{i}(t)$ exist; moreover, $\lim _{t \rightarrow t_{k}^{-}} \phi_{i}(t)=\phi_{i}\left(t_{k}\right)$ for $k=1,2, \ldots$, 
(3) $\phi_{i}(s)=\varphi_{i}(s)$ on $s \in\left[-m^{*}, 0\right]$,

(4) $\mathrm{e}^{\alpha t} \phi_{i}(t) \rightarrow 0$ as $t \rightarrow \infty$, where $\alpha=$ const and $0<$ $\alpha<\min _{i \in \mathcal{N}}\left\{a_{i}\right\}$,

where $t_{k}$ and $\varphi_{i}(s)$ are defined as shown in Section 2. Also $\mathscr{H}$ is a complete metric space when it is equipped with a metric defined by

$$
d(\overline{\mathbf{q}}(t), \overline{\mathbf{h}}(t))=\sum_{i=1}^{n} \sup _{t \geq-m^{*}}\left|q_{i}(t)-h_{i}(t)\right|,
$$

where $\overline{\mathbf{q}}(t)=\left(q_{1}(t), \ldots, q_{n}(t)\right) \in \mathscr{H}$ and $\overline{\mathbf{h}}(t)=$ $\left(h_{1}(t), \ldots, h_{n}(t)\right) \in \mathscr{H}$.

Theorem 3. Assume that conditions (A1)-(A4) hold provided that

(i) there exists a constant $\mu$ such that $\inf _{k=1,2, \ldots}\left\{t_{k}-t_{k-1}\right\} \geq$ $\mu$,

(ii) there exist constants $p_{i}$ such that $p_{i k} \leq p_{i} \mu$ for $i \in \mathcal{N}$ and $k=1,2, \ldots$,

(iii) $\sum_{i=1}^{n}\left\{\left(1 / a_{i}\right) \max _{j \in \mathcal{N}}\left|b_{i j} l_{j}\right|+\left(1 / a_{i}\right) \max _{j \in \mathcal{N}}\left|c_{i j} k_{j}\right|+\right.$ $\left.\left(\rho / a_{i}\right) \max _{j \in \mathcal{N}}\left|\omega_{j} d_{i j}\right|\right\}+\max _{i \in \mathcal{N}}\left\{p_{i}\left(\mu+\left(1 / a_{i}\right)\right)\right\} \triangleq \chi<$ 1 ,

and then the trivial equilibrium $\mathbf{x}=0$ is globally exponentially stable.

Proof. Multiplying both sides of (1) with $\mathrm{e}^{a_{i} t}$ gives, for $t>0$ and $t \neq t_{k}$,

$$
\begin{aligned}
\operatorname{de}^{a_{i} t} x_{i}(t)= & \mathrm{e}^{a_{i} t} \mathrm{~d} x_{i}(t)+a_{i} x_{i}(t) \mathrm{e}^{a_{i} t} \mathrm{~d} t \\
= & \mathrm{e}^{a_{i} t}\left\{\sum_{j=1}^{n} b_{i j} f_{j}\left(x_{j}(t)\right)+\sum_{j=1}^{n} c_{i j} g_{j}\left(x_{j}\left(t-\tau_{i j}(t)\right)\right)\right. \\
& \left.+\sum_{j=1}^{n} d_{i j} \int_{0}^{\rho(t)} \sigma_{j}\left(x_{j}(t-\theta)\right) \mathrm{d} \theta\right\} \mathrm{d} t,
\end{aligned}
$$

which yields after integrating from $t_{k-1}+\varepsilon(\varepsilon>0)$ to $t \epsilon$ $\left(t_{k-1}, t_{k}\right)(k=1,2, \ldots)$ that

$$
\begin{aligned}
& x_{i}(t) \mathrm{e}^{a_{i} t} \\
& =x_{i}\left(t_{k-1}+\varepsilon\right) \mathrm{e}^{a_{i}\left(t_{k-1}+\varepsilon\right)} \\
& \quad+\int_{t_{k-1}+\varepsilon}^{t} \mathrm{e}^{a_{i} s}\left\{\begin{array}{c}
\sum_{j=1}^{n} b_{i j} f_{j}\left(x_{j}(s)\right)+\sum_{j=1}^{n} c_{i j} g_{j}\left(x_{j}\left(s-\tau_{i j}(s)\right)\right) \\
+\sum_{j=1}^{n} d_{i j} \int_{0}^{\rho(s)} \sigma_{j}\left(x_{j}(s-\theta)\right) \mathrm{d} \theta
\end{array}\right\} \mathrm{d} s .
\end{aligned}
$$

Letting $\varepsilon \rightarrow 0$ in (12), we have, for $t \in\left(t_{k-1}, t_{k}\right)(k=$ $1,2, \ldots)$

$$
\begin{aligned}
& x_{i}(t) \mathrm{e}^{a_{i} t} \\
& =x_{i}\left(t_{k-1}+0\right) \mathrm{e}^{a_{i} t_{k-1}} \\
& \quad+\int_{t_{k-1}}^{t} \mathrm{e}^{a_{i} s}\left\{\begin{array}{c}
\sum_{j=1}^{n} b_{i j} f_{j}\left(x_{j}(s)\right)+\sum_{j=1}^{n} c_{i j} g_{j}\left(x_{j}\left(s-\tau_{i j}(s)\right)\right) \\
+\sum_{j=1}^{n} d_{i j} \int_{0}^{\rho(s)} \sigma_{j}\left(x_{j}(s-\theta)\right) \mathrm{d} \theta
\end{array}\right\} \mathrm{d} s .
\end{aligned}
$$

Setting $t=t_{k}-\varepsilon(\varepsilon>0)$ in (13), we get

$$
\begin{aligned}
& x_{i}\left(t_{k}-\varepsilon\right) \mathrm{e}^{a_{i}\left(t_{k}-\varepsilon\right)} \\
& =x_{i}\left(t_{k-1}+0\right) \mathrm{e}^{a_{i} t_{k-1}} \\
& \quad+\int_{t_{k-1}}^{t_{k}-\varepsilon} \mathrm{e}^{a_{i} s}\left\{\begin{array}{c}
\sum_{j=1}^{n} b_{i j} f_{j}\left(x_{j}(s)\right)+\sum_{j=1}^{n} c_{i j} g_{j}\left(x_{j}\left(s-\tau_{i j}(s)\right)\right) \\
+\sum_{j=1}^{n} d_{i j} \int_{0}^{\rho(s)} \sigma_{j}\left(x_{j}(s-\theta)\right) \mathrm{d} \theta
\end{array}\right\} \mathrm{d} s,
\end{aligned}
$$

which generates by letting $\varepsilon \rightarrow 0$

$$
\begin{aligned}
& x_{i}\left(t_{k}-0\right) \mathrm{e}^{a_{i} t_{k}} \\
& =x_{i}\left(t_{k-1}+0\right) \mathrm{e}^{a_{i} t_{k-1}} \\
& \quad+\int_{t_{k-1}}^{t_{k}} \mathrm{e}^{a_{i} s}\left\{\begin{array}{c}
\sum_{j=1}^{n} b_{i j} f_{j}\left(x_{j}(s)\right)+\sum_{j=1}^{n} c_{i j} g_{j}\left(x_{j}\left(s-\tau_{i j}(s)\right)\right) \\
+\sum_{j=1}^{n} d_{i j} \int_{0}^{\rho(s)} \sigma_{j}\left(x_{j}(s-\theta)\right) \mathrm{d} \theta
\end{array}\right\} \mathrm{d} s .
\end{aligned}
$$

Noting $x_{i}\left(t_{k}-0\right)=x_{i}\left(t_{k}\right)$, (15) can be rearranged as

$$
\begin{aligned}
& x_{i}\left(t_{k}\right) \mathrm{e}^{a_{i} t_{k}} \\
& =x_{i}\left(t_{k-1}+0\right) \mathrm{e}^{a_{i} t_{k-1}} \\
& \quad+\int_{t_{k-1}}^{t_{k}} \mathrm{e}^{a_{i} s}\left\{\begin{array}{c}
\sum_{j=1}^{n} b_{i j} f_{j}\left(x_{j}(s)\right)+\sum_{j=1}^{n} c_{i j} g_{j}\left(x_{j}\left(s-\tau_{i j}(s)\right)\right) \\
+\sum_{j=1}^{n} d_{i j} \int_{0}^{\rho(s)} \sigma_{j}\left(x_{j}(s-\theta)\right) \mathrm{d} \theta
\end{array}\right\} \mathrm{d} s .
\end{aligned}
$$

Combining (13) and (16), we derive that

$$
\begin{aligned}
& x_{i}(t) \mathrm{e}^{a_{i} t} \\
& =x_{i}\left(t_{k-1}+0\right) \mathrm{e}^{a_{i} t_{k-1}} \\
& \quad+\int_{t_{k-1}}^{t} \mathrm{e}^{a_{i} s}\left\{\begin{array}{c}
\sum_{j=1}^{n} b_{i j} f_{j}\left(x_{j}(s)\right)+\sum_{j=1}^{n} c_{i j} g_{j}\left(x_{j}\left(s-\tau_{i j}(s)\right)\right) \\
+\sum_{j=1}^{n} d_{i j} \int_{0}^{\rho(s)} \sigma_{j}\left(x_{j}(s-\theta)\right) \mathrm{d} \theta
\end{array}\right\} \mathrm{d} s
\end{aligned}
$$


is true for $t \in\left(t_{k-1}, t_{k}\right](k=1,2, \ldots)$. Hence, we get, for $t \in$ $\left(t_{k-1}, t_{k}\right](k=1,2, \ldots)$,

$$
\begin{aligned}
& x_{i}(t) \mathrm{e}^{a_{i} t} \\
& =\left\{x_{i}\left(t_{k-1}\right)+I_{i(k-1)}\left(x_{i}\left(t_{k-1}\right)\right)\right\} \mathrm{e}^{a_{i} t_{k-1}} \\
& \quad+\int_{t_{k-1}}^{t} \mathrm{e}^{a_{i} s}\left\{\begin{array}{c}
\sum_{j=1}^{n} b_{i j} f_{j}\left(x_{j}(s)\right)+\sum_{j=1}^{n} c_{i j} g_{j}\left(x_{j}\left(s-\tau_{i j}(s)\right)\right) \\
+\sum_{j=1}^{n} d_{i j} \int_{0}^{\rho(s)} \sigma_{j}\left(x_{j}(s-\theta)\right) \mathrm{d} \theta
\end{array}\right\} \mathrm{d} s \\
& =x_{i}\left(t_{k-1}\right) \mathrm{e}^{a_{i} t_{k-1}} \\
& +\int_{t_{k-1}}^{t} \mathrm{e}^{a_{i} s}\left\{\begin{array}{c}
\sum_{j=1}^{n} b_{i j} f_{j}\left(x_{j}(s)\right)+\sum_{j=1}^{n} c_{i j} g_{j}\left(x_{j}\left(s-\tau_{i j}(s)\right)\right) \\
+\sum_{j=1}^{n} d_{i j} \int_{0}^{\rho(s)} \sigma_{j}\left(x_{j}(s-\theta)\right) \mathrm{d} \theta
\end{array}\right\} \mathrm{d} s \\
& +I_{i(k-1)}\left(x_{i}\left(t_{k-1}\right)\right) \mathrm{e}^{a_{i} t_{k-1}},
\end{aligned}
$$

which results in

$$
\begin{aligned}
& x_{i}\left(t_{k-1}\right) \mathrm{e}^{a_{i} t_{k-1}} \\
& =x_{i}\left(t_{k-2}\right) \mathrm{e}^{a_{i} t_{k-2}} \\
& +\int_{t_{k-2}}^{t_{k-1}} \mathrm{e}^{a_{i} s}\left\{\begin{array}{c}
\sum_{j=1}^{n} b_{i j} f_{j}\left(x_{j}(s)\right)+\sum_{j=1}^{n} c_{i j} g_{j}\left(x_{j}\left(s-\tau_{i j}(s)\right)\right) \\
+\sum_{j=1}^{n} d_{i j} \int_{0}^{\rho(s)} \sigma_{j}\left(x_{j}(s-\theta)\right) \mathrm{d} \theta
\end{array}\right\} \mathrm{d} s \\
& +I_{i(k-2)}\left(x_{i}\left(t_{k-2}\right)\right) \mathrm{e}^{a_{i} t_{k-2}} \\
& x_{i}\left(t_{2}\right) \mathrm{e}^{a_{i} t_{2}} \\
& =x_{i}\left(t_{1}\right) \mathrm{e}^{a_{i} t_{1}} \\
& +\int_{t_{1}}^{t_{2}} \mathrm{e}^{a_{i} s}\left\{\begin{array}{c}
\sum_{j=1}^{n} b_{i j} f_{j}\left(x_{j}(s)\right)+\sum_{j=1}^{n} c_{i j} g_{j}\left(x_{j}\left(s-\tau_{i j}(s)\right)\right) \\
+\sum_{j=1}^{n} d_{i j} \int_{0}^{\rho(s)} \sigma_{j}\left(x_{j}(s-\theta)\right) \mathrm{d} \theta
\end{array}\right\} \mathrm{d} s \\
& +I_{i 1}\left(x_{i}\left(t_{1}\right)\right) \mathrm{e}^{a_{i} t_{1}}, \\
& x_{i}\left(t_{1}\right) \mathrm{e}^{a_{i} t_{1}} \\
& =\varphi_{i}(0) \\
& +\int_{0}^{t_{1}} \mathrm{e}^{a_{i} s}\left\{\begin{array}{c}
\sum_{j=1}^{n} b_{i j} f_{j}\left(x_{j}(s)\right)+\sum_{j=1}^{n} c_{i j} g_{j}\left(x_{j}\left(s-\tau_{i j}(s)\right)\right) \\
+\sum_{j=1}^{n} d_{i j} \int_{0}^{\rho(s)} \sigma_{j}\left(x_{j}(s-\theta)\right) \mathrm{d} \theta
\end{array}\right\} \mathrm{d} s .
\end{aligned}
$$

We therefore conclude, for $t>0$,

$$
\begin{aligned}
& x_{i}(t) \\
& =\varphi_{i}(0) \mathrm{e}^{-a_{i} t} \\
& +\mathrm{e}^{-a_{i} t} \int_{0}^{t} \mathrm{e}^{a_{i} s}\left\{\begin{array}{c}
\sum_{j=1}^{n} b_{i j} f_{j}\left(x_{j}(s)\right)+\sum_{j=1}^{n} c_{i j} g_{j}\left(x_{j}\left(s-\tau_{i j}(s)\right)\right) \\
+\sum_{j=1}^{n} d_{i j} \int_{0}^{\rho(s)} \sigma_{j}\left(x_{j}(s-\theta)\right) \mathrm{d} \theta
\end{array}\right\} \mathrm{d} s \\
& +\mathrm{e}^{-a_{i} t} \sum_{0<t_{k}<t}\left\{I_{i k}\left(x_{i}\left(t_{k}\right)\right) \mathrm{e}^{a_{i} t_{k}}\right\} .
\end{aligned}
$$

Note that $x_{i}(0)=\varphi_{i}(0)$ in $(20)$. We then define the following operator $\pi$ acting on $\mathscr{H}$, for $\overline{\mathbf{y}}(t)=\left(y_{1}(t), \ldots, y_{n}(t)\right) \in \mathscr{H}$ :

$$
\pi(\overline{\mathbf{y}})(t)=\left(\pi\left(y_{1}\right)(t), \ldots, \pi\left(y_{n}\right)(t)\right),
$$

where $\pi\left(y_{i}\right)(t):\left[-m^{*},+\infty\right) \rightarrow R(i \in \mathcal{N})$ obeys the rule as follows:

$$
\begin{aligned}
& \pi\left(y_{i}\right)(t) \\
& =\varphi_{i}(0) \mathrm{e}^{-a_{i} t} \\
& +\mathrm{e}^{-a_{i} t} \int_{0}^{t} \mathrm{e}^{a_{i} s}\left\{\begin{array}{c}
\sum_{j=1}^{n} b_{i j} f_{j}\left(y_{j}(s)\right)+\sum_{j=1}^{n} c_{i j} g_{j}\left(y_{j}\left(s-\tau_{i j}(s)\right)\right) \\
+\sum_{j=1}^{n} d_{i j} \int_{0}^{\rho(s)} \sigma_{j}\left(y_{j}(s-\theta)\right) \mathrm{d} \theta
\end{array}\right\} \mathrm{d} s \\
& +\mathrm{e}^{-a_{i} t} \sum_{0<t_{k}<t}\left\{I_{i k}\left(y_{i}\left(t_{k}\right)\right) \mathrm{e}^{a_{i} t_{k}}\right\}
\end{aligned}
$$

on $t \geq 0$ and $\pi\left(y_{i}\right)(s)=\varphi_{i}(s)$ on $s \in\left[-m^{*}, 0\right]$.

In what follows, we will apply the contraction mapping principle to prove the existence and uniqueness of solution and the global exponential stability of trivial equilibrium at the same time. The subsequent proof can be divided into two steps.

Step 1. We need to prove that $\pi(\mathscr{H}) \subset \mathscr{H}$. For $y_{i}(t) \in \mathscr{H}_{i}$ $(i \in \mathcal{N})$, it is necessary to show that $\pi\left(y_{i}\right)(t) \subset \mathscr{H}_{i}$. As defined above, we see that $\pi\left(y_{i}\right)(s)=\varphi_{i}(s)$ on $s \in\left[-m^{*}, 0\right]$. Owing to the continuity of $\varphi_{i}(s)$ on $s \in\left[-m^{*}, 0\right]$, we immediately know that $\pi\left(y_{i}\right)(t)$ is continuous on $t \in\left[-m^{*}, 0\right]$.

Choose a fixed time $t>0$, and it is then derived from (22) that

$\pi\left(y_{i}\right)(t+r)-\pi\left(y_{i}\right)(t)=Q_{1}+Q_{2}+Q_{3}+Q_{4}+Q_{5}, \quad t>0$,

where,

$$
\begin{gathered}
Q_{1}=\varphi_{i}(0) \mathrm{e}^{-a_{i}(t+r)}-\varphi_{i}(0) \mathrm{e}^{-a_{i} t}, \\
Q_{2}=\mathrm{e}^{-a_{i}(t+r)} \int_{0}^{t+r} \mathrm{e}^{a_{i} s} \sum_{j=1}^{n} b_{i j} f_{j}\left(y_{j}(s)\right) \mathrm{d} s \\
-\mathrm{e}^{-a_{i} t} \int_{0}^{t} \mathrm{e}^{a_{i} s} \sum_{j=1}^{n} b_{i j} f_{j}\left(y_{j}(s)\right) \mathrm{d} s,
\end{gathered}
$$




$$
\begin{gathered}
Q_{3}=\mathrm{e}^{-a_{i}(t+r)} \int_{0}^{t+r} \mathrm{e}^{a_{i} s} \sum_{j=1}^{n} c_{i j} g_{j}\left(y_{j}\left(s-\tau_{i j}(s)\right)\right) \mathrm{d} s \\
-\mathrm{e}^{-a_{i} t} \int_{0}^{t} \mathrm{e}^{a_{i} s} \sum_{j=1}^{n} c_{i j} g_{j}\left(y_{j}\left(s-\tau_{i j}(s)\right)\right) \mathrm{d} s, \\
Q_{4}=\mathrm{e}^{-a_{i}(t+r)} \int_{0}^{t+r} \mathrm{e}^{a_{i} s} \sum_{j=1}^{n} d_{i j} \int_{0}^{\rho(s)} \sigma_{j}\left(y_{j}(s-\theta)\right) \mathrm{d} \theta \mathrm{d} s \\
-\mathrm{e}^{-a_{i} t} \int_{0}^{t} \mathrm{e}^{a_{i} s} \sum_{j=1}^{n} d_{i j} \int_{0}^{\rho(s)} \sigma_{j}\left(y_{j}(s-\theta)\right) \mathrm{d} \theta \mathrm{d} s, \\
Q_{5}=\mathrm{e}^{-a_{i}(t+r)} \sum_{0<t_{k}<(t+r)}\left\{I_{i k}\left(y_{i}\left(t_{k}\right)\right) \mathrm{e}^{a_{i} t_{k}}\right\} \\
-\mathrm{e}^{-a_{i} t} \sum_{0<t_{k}<t}\left\{I_{i k}\left(y_{i}\left(t_{k}\right)\right) \mathrm{e}^{a_{i} t_{k}}\right\} .
\end{gathered}
$$

Since $y_{i}(t) \in \mathscr{H}_{i}$, we know that $y_{i}(t)$ is continuous on $t \neq t_{k}(k=1,2, \ldots)$; moreover, $\lim _{t \rightarrow t_{k}^{-}} y_{i}(t)$ and $\lim _{t \rightarrow t_{k}^{+}} y_{i}(t)$ exist, in addition, $\lim _{t \rightarrow t_{k}^{-}} y_{i}(t)=y_{i}\left(t_{k}\right)$.

Letting $t \neq t_{k}(k=1,2, \ldots)$ in $(23)$, it is easy to see that $Q_{i} \rightarrow 0$ as $r \rightarrow 0$ for $i=1, \ldots, 5$. Thus, $\pi\left(y_{i}\right)(t+r)-$ $\pi\left(y_{i}\right)(t) \rightarrow 0$ as $r \rightarrow 0$ holds on $t>0$ and $t \neq t_{k}(k=$ $1,2, \ldots)$.

Letting $t=t_{k}(k=1,2, \ldots)$ in (23), it is not difficult to find that $Q_{i} \rightarrow 0$ as $r \rightarrow 0$ for $i=1, \ldots, 4$. Letting $r<0$ be small enough, we compute

$$
\begin{aligned}
Q_{5}= & \mathrm{e}^{-a_{i}\left(t_{k}+r\right)} \sum_{0<t_{m}<\left(t_{k}+r\right)} I_{i m}\left(y_{i}\left(t_{m}\right)\right) \mathrm{e}^{a_{i} t_{m}} \\
& -\mathrm{e}^{-a_{i} t_{k}} \sum_{0<t_{m}<t_{k}} I_{i m}\left(y_{i}\left(t_{m}\right)\right) \mathrm{e}^{a_{i} t_{m}} \\
= & \left\{\mathrm{e}^{-a_{i}\left(t_{k}+r\right)}-\mathrm{e}^{-a_{i} t_{k}}\right\} \sum_{0<t_{m}<t_{k}}\left\{I_{i m}\left(y_{i}\left(t_{m}\right)\right) \mathrm{e}^{a_{i} t_{m}}\right\}
\end{aligned}
$$

which implies $\lim _{r \rightarrow 0^{-}} Q_{5}=0$. Letting $r>0$ be small enough, we have

$$
\begin{aligned}
Q_{5}= & \mathrm{e}^{-a_{i}\left(t_{k}+r\right)} \sum_{0<t_{m}<\left(t_{k}+r\right)} I_{i m}\left(y_{i}\left(t_{m}\right)\right) \mathrm{e}^{a_{i} t_{m}} \\
& -\mathrm{e}^{-a_{i} t_{k}} \sum_{0<t_{m}<t_{k}} I_{i m}\left(y_{i}\left(t_{m}\right)\right) \mathrm{e}^{a_{i} t_{m}} \\
= & \mathrm{e}^{-a_{i}\left(t_{k}+r\right)}\left\{\sum_{0<t_{m}<t_{k}}\left\{I_{i m}\left(y_{i}\left(t_{m}\right)\right) \mathrm{e}^{a_{i} t_{m}}\right\}+I_{i k}\left(y_{i}\left(t_{k}\right)\right) \mathrm{e}^{a_{i} t_{k}}\right\} \\
& -\mathrm{e}^{-a_{i} t_{k}} \sum_{0<t_{m}<t_{k}}\left\{I_{i m}\left(y_{i}\left(t_{m}\right)\right) \mathrm{e}^{a_{i} t_{m}}\right\} \\
= & \left\{\mathrm{e}^{-a_{i}\left(t_{k}+r\right)}-\mathrm{e}^{-a_{i} t_{k}}\right\} \sum_{0<t_{m}<t_{k}}\left\{I_{i m}\left(y_{i}\left(t_{m}\right)\right) \mathrm{e}^{a_{i} t_{m}}\right\} \\
& +\mathrm{e}^{-a_{i}\left(t_{k}+r\right)} I_{i k}\left(y_{i}\left(t_{k}\right)\right) \mathrm{e}^{a_{i} t_{k}},
\end{aligned}
$$

which implies $\lim _{r \rightarrow 0^{+}} Q_{5}=\mathrm{e}^{-a_{i} t_{k}} I_{i k}\left(y_{i}\left(t_{k}\right)\right) \mathrm{e}^{a_{i} t_{k}}$.
According to the above discussion, we see that $\pi\left(y_{i}\right)(t)$ : $\left[-m^{*},+\infty\right) \rightarrow R$ is continuous on $t \neq t_{k}(k=1,2, \ldots)$, while for $t=t_{k}(k=1,2, \ldots), \lim _{t \rightarrow t_{k}^{-}} \pi\left(y_{i}\right)(t)$ and $\lim _{t \rightarrow t_{k}^{+}} \pi\left(y_{i}\right)(t)$ exist; moreover, $\lim _{t \rightarrow t_{k}^{-}} \pi\left(y_{i}\right)(t)=$ $\pi\left(y_{i}\right)\left(t_{k}\right) \neq \lim _{t \rightarrow t_{k}^{+}} \pi\left(y_{i}\right)(t)$.

Next, we will prove that $\mathrm{e}^{\alpha t} \pi\left(y_{i}\right)(t) \rightarrow 0$ as $t \rightarrow \infty$ for $i \in \mathcal{N}$. To begin with, we give the expression of $\mathrm{e}^{\alpha t} \pi\left(y_{i}\right)(t)$ as follows:

$$
\mathrm{e}^{\alpha t} \pi\left(y_{i}\right)(t)=W_{1}+W_{2}+W_{3}+W_{4}+W_{5}, \quad t>0,
$$

where

$$
\begin{aligned}
& W_{1}=\varphi_{i}(0) \mathrm{e}^{-\left(a_{i}-\alpha\right) t}, \\
& W_{2}=\mathrm{e}^{\alpha t} \mathrm{e}^{-a_{i} t} \int_{0}^{t} \mathrm{e}^{a_{i} s} \sum_{j=1}^{n} b_{i j} f_{j}\left(y_{j}(s)\right) \mathrm{d} s, \\
& W_{5}=\mathrm{e}^{\alpha t} \mathrm{e}^{-a_{i} t} \sum_{0<t_{k}<t}\left\{I_{i k}\left(y_{i}\left(t_{k}\right)\right) \mathrm{e}^{a_{i} t_{k}}\right\}, \\
& W_{3}=\mathrm{e}^{\alpha t} \mathrm{e}^{-a_{i} t} \int_{0}^{t} \mathrm{e}^{a_{i} s} \sum_{j=1}^{n} c_{i j} g_{j}\left(y_{j}\left(s-\tau_{i j}(s)\right)\right) \mathrm{d} s, \text { and } \\
& W_{4}=\mathrm{e}^{\alpha t} \mathrm{e}^{-a_{i} t} \int_{0}^{t} \mathrm{e}^{a_{i} s} \sum_{j=1}^{n} d_{i j} \int_{0}^{\rho(s)} \sigma_{j}\left(y_{j}(s-\theta)\right) \mathrm{d} \theta \mathrm{d} s .
\end{aligned}
$$

First, it is obvious that $\lim _{t \rightarrow \infty} W_{1}=0$ as $a_{i}-\alpha>$ 0 . Furthermore, for $y_{j}(t) \in \mathscr{H}_{j}(j \in \mathcal{N})$, we see $\lim _{t \rightarrow \infty} \mathrm{e}^{\alpha t} y_{j}(t)=0$. Then, for any $\varepsilon>0$, there exists a $T_{j}>0$ such that $s \geq T_{j}$ implies $\left|\mathrm{e}^{\alpha s} y_{j}(s)\right|<\varepsilon$. Choose $T^{*}=\max _{j \in \mathscr{N}}\left\{T_{j}\right\}$. It is derived form (A1) that

$$
\begin{aligned}
W_{2} \leq & \mathrm{e}^{\alpha t} \mathrm{e}^{-a_{i} t} \int_{0}^{t} \mathrm{e}^{a_{i} s} \sum_{j=1}^{n}\left\{\left|b_{i j} l_{j}\right|\left|y_{j}(s)\right|\right\} \mathrm{d} s \\
= & \mathrm{e}^{\alpha t} \mathrm{e}^{-a_{i} t} \int_{0}^{t} \mathrm{e}^{a_{i} s} \mathrm{e}^{-\alpha s} \sum_{j=1}^{n}\left\{\left|b_{i j} l_{j}\right| \mathrm{e}^{\alpha s}\left|y_{j}(s)\right|\right\} \mathrm{d} s \\
= & \mathrm{e}^{-\left(a_{i}-\alpha\right) t} \int_{0}^{T^{*}} \mathrm{e}^{\left(a_{i}-\alpha\right) s} \sum_{j=1}^{n}\left\{\left|b_{i j} l_{j}\right| \mathrm{e}^{\alpha s}\left|y_{j}(s)\right|\right\} \mathrm{d} s \\
& +\mathrm{e}^{-\left(a_{i}-\alpha\right) t} \int_{T^{*}}^{t} \mathrm{e}^{\left(a_{i}-\alpha\right) s} \sum_{j=1}^{n}\left\{\left|b_{i j} l_{j}\right| \mathrm{e}^{\alpha s}\left|y_{j}(s)\right|\right\} \mathrm{d} s \\
\leq & \mathrm{e}^{-\left(a_{i}-\alpha\right) t} \sum_{j=1}^{n}\left\{\left|b_{i j} l_{j}\right| \sup _{s \in\left[0, T^{*}\right]}\left|\mathrm{e}^{\alpha s} y_{j}(s)\right|\right\}\left\{\int_{0}^{T^{*}} \mathrm{e}^{\left(a_{i}-\alpha\right) s} \mathrm{~d} s\right\} \\
& +\frac{\varepsilon}{a_{i}-\alpha} \sum_{j=1}^{n}\left\{\left|b_{i j} l_{j}\right|\right\} \mathrm{e}^{-\left(a_{i}-\alpha\right) t} \int_{T^{*}}^{t} \mathrm{e}^{\left(a_{i}-\alpha\right) s} \mathrm{~d} s \\
\leq & \left.\mathrm{e}^{-\left(a_{i}-\alpha\right) t} \sum_{j=1}^{n}\left\{\left|b_{i j} l_{i j} l_{j}\right|\right\} \sup _{s \in\left[0, T^{*}\right]}\left|\mathrm{e}^{\alpha s} y_{j}(s)\right|\right\}\left\{\int_{0}^{T^{*}} \mathrm{e}^{\left(a_{i}-\alpha\right) s} \mathrm{~d} s\right\}
\end{aligned}
$$

which leads to $W_{2} \rightarrow 0$ as $t \rightarrow \infty$. 
Similarly, for the given $\varepsilon>0$ above, there also exists a $T_{j}^{\prime}>0$ such that $s \geq T_{j}^{\prime}-\tau$ implies $\left|\mathrm{e}^{\alpha s} y_{j}(s)\right|<\varepsilon$. Select $\widehat{T}=\max _{j \in \mathcal{N}}\left\{T_{j}^{\prime}\right\}$. It follows from (A2) that

$$
\begin{aligned}
& W_{3} \leq \mathrm{e}^{\alpha t} \mathrm{e}^{-a_{i} t} \int_{0}^{t} \mathrm{e}^{a_{i} s} \sum_{j=1}^{n}\left\{\left|c_{i j} k_{j}\right|\left|y_{j}\left(s-\tau_{i j}(s)\right)\right|\right\} \mathrm{d} s \\
& \leq \mathrm{e}^{-\left(a_{i}-\alpha\right) t} \\
& \times \int_{0}^{t} \mathrm{e}^{a_{i} s} \mathrm{e}^{-\alpha\{s-\tau\}} \\
& \times \sum_{j=1}^{n}\left\{\left|c_{i j} k_{j}\right| \mathrm{e}^{\alpha\left[s-\tau_{i j}(s)\right]}\left|y_{j}\left(s-\tau_{i j}(s)\right)\right|\right\} \mathrm{d} s \\
& =\mathrm{e}^{\alpha \tau} \mathrm{e}^{-\left(a_{i}-\alpha\right) t} \\
& \times \int_{0}^{\widehat{T}} \mathrm{e}^{\left(a_{i}-\alpha\right) s} \sum_{j=1}^{n}\left\{\left|c_{i j} k_{j}\right| \mathrm{e}^{\alpha\left[s-\tau_{i j}(s)\right]}\left|y_{j}\left(s-\tau_{i j}(s)\right)\right|\right\} \mathrm{d} s \\
& +\mathrm{e}^{\alpha \tau} \mathrm{e}^{-\left(a_{i}-\alpha\right) t} \\
& \times \int_{\widehat{T}}^{t} \mathrm{e}^{\left(a_{i}-\alpha\right) s} \sum_{j=1}^{n}\left\{\left|c_{i j} k_{j}\right| \mathrm{e}^{\alpha\left[s-\tau_{i j}(s)\right]}\left|y_{j}\left(s-\tau_{i j}(s)\right)\right|\right\} \mathrm{d} s \\
& \leq \mathrm{e}^{\alpha \tau} \sum_{j=1}^{n}\left\{\left|c_{i j} k_{j}\right| \sup _{s \in[-\tau, \widehat{T}]}\left|\mathrm{e}^{\alpha s} y_{j}(s)\right|\right\} \\
& \times \mathrm{e}^{-\left(a_{i}-\alpha\right) t} \int_{0}^{\widehat{T}} \mathrm{e}^{\left(a_{i}-\alpha\right) s} \mathrm{~d} s \\
& +\mathrm{e}^{\alpha \tau} \varepsilon \sum_{j=1}^{n}\left\{\left|c_{i j} k_{j}\right|\right\} \mathrm{e}^{-\left(a_{i}-\alpha\right) t} \int_{\widehat{T}}^{t} \mathrm{e}^{\left(a_{i}-\alpha\right) s} \mathrm{~d} s \\
& \leq \mathrm{e}^{\alpha \tau} \sum_{j=1}^{n}\left\{\left|c_{i j} k_{j}\right| \sup _{s \in[-\tau, \widehat{T}]}\left|e^{\alpha s} y_{j}(s)\right|\right\} \\
& \times \mathrm{e}^{-\left(a_{i}-\alpha\right) t} \int_{0}^{\widehat{T}} \mathrm{e}^{\left(a_{i}-\alpha\right) s} \mathrm{~d} s+\frac{\mathrm{e}^{\alpha \tau} \varepsilon}{a_{i}-\alpha} \sum_{j=1}^{n}\left\{\left|c_{i j} k_{j}\right|\right\},
\end{aligned}
$$

which results in $W_{3} \rightarrow 0$ as $t \rightarrow \infty$. In addition, it is derived from (A4) that

$$
\begin{aligned}
W_{4} \leq & \mathrm{e}^{\alpha t} \mathrm{e}^{-a_{i} t} \int_{0}^{t} \mathrm{e}^{a_{i} s} \sum_{j=1}^{n}\left\{d_{i j} \int_{0}^{\rho} \omega_{j}\left|y_{j}(s-\theta)\right| \mathrm{d} \theta\right\} \mathrm{d} s \\
= & \mathrm{e}^{\alpha t} \mathrm{e}^{-a_{i} t} \\
& \quad \times \int_{0}^{t} \mathrm{e}^{a_{i} s} \mathrm{e}^{-\alpha s} \sum_{j=1}^{n}\left\{d_{i j} \int_{0}^{\rho} \mathrm{e}^{\alpha \theta} \omega_{j} \mathrm{e}^{\alpha(s-\theta)}\left|y_{j}(s-\theta)\right| \mathrm{d} \theta\right\} \mathrm{d} s \\
\leq & \mathrm{e}^{\alpha t} \mathrm{e}^{-a_{i} t} \\
& \quad \int_{0}^{t} \mathrm{e}^{a_{i} s} \mathrm{e}^{-\alpha s} \\
& \quad \times \sum_{j=1}^{n}\left\{d_{i j} \sup _{\zeta \in[s-\rho, s]}\left\{\mathrm{e}^{\alpha \zeta}\left|y_{j}(\zeta)\right|\right\} \int_{0}^{\rho} \mathrm{e}^{\alpha \theta} \omega_{j} \mathrm{~d} \theta\right\} \mathrm{d} s .
\end{aligned}
$$

Since $\mathrm{e}^{\alpha \zeta}\left|y_{j}(\zeta)\right| \rightarrow 0$ as $\zeta \rightarrow \infty$, we know that, for any $\varepsilon>0$, there exists a $T_{j}^{\prime \prime}>0$ such that $\zeta>T_{j}^{\prime \prime}-\rho$ implies $\mathrm{e}^{\alpha \zeta}\left|y_{j}(\zeta)\right|<\varepsilon$. Selecting $\bar{T}=\max _{j \in \mathcal{N}}\left\{T_{j}^{\prime \prime}\right\}$, it follows from (30) that

$$
\begin{aligned}
& W_{4} \leq \mathrm{e}^{\left(\alpha-a_{i}\right) t} \\
& \times \int_{0}^{\bar{T}} \mathrm{e}^{\left(a_{i}-\alpha\right) s} \sum_{j=1}^{n}\left\{d_{i j} \sup _{\zeta \in[s-\rho, s]}\left\{\mathrm{e}^{\alpha \zeta}\left|y_{j}(\zeta)\right|\right\}\right. \\
& \left.\times \int_{0}^{\rho} \mathrm{e}^{\alpha \theta} \omega_{j} \mathrm{~d} \theta\right\} \mathrm{d} s \\
& +\mathrm{e}^{\left(\alpha-a_{i}\right) t} \\
& \times \int_{\bar{T}}^{t} \mathrm{e}^{\left(a_{i}-\alpha\right) s} \sum_{j=1}^{n}\left\{d_{i j} \sup _{\zeta \in[s-\rho, s]}\left\{\mathrm{e}^{\alpha \zeta}\left|y_{j}(\zeta)\right|\right\}\right. \\
& \left.\times \int_{0}^{\rho} e^{\alpha \theta} \omega_{j} d \theta\right\} d s \\
& \leq \frac{\mathrm{e}^{\alpha \rho}}{\alpha}\left\{\sum_{j=1}^{n} d_{i j} \omega_{j} \sup _{\zeta \in[-\rho, \bar{T}]}\left\{\mathrm{e}^{\alpha \zeta}\left|y_{j}(\zeta)\right|\right\}\right\} \\
& \times \mathrm{e}^{\left(\alpha-a_{i}\right) t} \int_{0}^{\bar{T}} \mathrm{e}^{\left(a_{i}-\alpha\right) s} \mathrm{~d} s \\
& +\frac{\mathrm{e}^{\alpha \rho}}{\alpha} \sum_{j=1}^{n}\left\{d_{i j} \omega_{j} \sup _{\zeta \in[\bar{T}-\rho, t]}\left\{\mathrm{e}^{\alpha \zeta}\left|y_{j}(\zeta)\right|\right\}\right\} \\
& \times \mathrm{e}^{\left(\alpha-a_{i}\right) t} \int_{\bar{T}}^{t} \mathrm{e}^{\left(a_{i}-\alpha\right) s} \mathrm{~d} s \\
& \leq \mathrm{e}^{\left(\alpha-a_{i}\right) t} \frac{e^{\alpha \rho}}{\alpha}\left\{\sum_{j=1}^{n} d_{i j} \omega_{j} \sup _{\zeta \in[-\rho, \bar{T}]}\left\{\mathrm{e}^{\alpha \zeta}\left|y_{j}(\zeta)\right|\right\}\right\} \\
& \times \int_{0}^{\bar{T}} \mathrm{e}^{\left(a_{i}-\alpha\right) s} \mathrm{~d} s+\varepsilon \sum_{j=1}^{n}\left\{d_{i j} \omega_{j}\right\} \frac{\mathrm{e}^{\alpha \rho}}{\alpha\left(a_{i}-\alpha\right)},
\end{aligned}
$$

which yields $W_{4} \rightarrow 0$ as $t \rightarrow \infty$.

Furthermore, from (A3), we see that $\left|I_{i k}\left(x_{i}\left(t_{k}\right)\right)\right| \leq$ $p_{i k}\left|y_{i}\left(t_{k}\right)\right|$. So,

$$
W_{5} \leq \mathrm{e}^{\alpha t} \mathrm{e}^{-a_{i} t} \sum_{0<t_{k}<t}\left\{p_{i k}\left|y_{i}\left(t_{k}\right)\right| \mathrm{e}^{a_{i} t_{k}}\right\} .
$$

As $y_{i}(t) \in \mathscr{H}_{i}$, we have $\lim _{t \rightarrow \infty} \mathrm{e}^{\alpha t} y_{i}(t)=0$. Then, for any $\varepsilon>0$, there exists a nonimpulsive point $T_{i}>0$ such that 
$s \geq T_{i}$ implies $\left|\mathrm{e}^{\alpha s} y_{i}(s)\right|<\varepsilon$. It then follows from conditions (i) and (ii) that

$$
\begin{aligned}
& W_{5} \leq \mathrm{e}^{\alpha t} \mathrm{e}^{-a_{i} t}\left\{\sum_{0<t_{k}<T_{i}}\left\{p_{i k}\left|y_{i}\left(t_{k}\right)\right| \mathrm{e}^{a_{i} t_{k}}\right\}\right. \\
& \left.+\sum_{T_{i}<t_{k}<t}\left\{p_{i k}\left|y_{i}\left(t_{k}\right)\right| \mathrm{e}^{\alpha t_{k}} \mathrm{e}^{\left(a_{i}-\alpha\right) t_{k}}\right\}\right\} \\
& \leq \mathrm{e}^{\alpha t} \mathrm{e}^{-a_{i} t} \sum_{0<t_{k}<T_{i}}\left\{p_{i k}\left|y_{i}\left(t_{k}\right)\right| \mathrm{e}^{a_{i} t_{k}}\right\} \\
& +\mathrm{e}^{\alpha t} \mathrm{e}^{-a_{i} t} p_{i} \varepsilon \sum_{T_{i}<t_{k}<t}\left\{\mu \mathrm{e}^{\left(a_{i}-\alpha\right) t_{k}}\right\} \\
& \leq \mathrm{e}^{-\left(a_{i}-\alpha\right) t} \sum_{0<t_{k}<T_{i}}\left\{p_{i k}\left|y_{i}\left(t_{k}\right)\right| \mathrm{e}^{a_{i} t_{k}}\right\} \\
& +\mathrm{e}^{-\left(a_{i}-\alpha\right) t} p_{i} \varepsilon\left\{\sum_{T_{i}<t_{r}<t_{k}}\left\{\mathrm{e}^{\left(a_{i}-\alpha\right) t_{r}}\left(t_{r+1}-t_{r}\right)\right\}\right. \\
& \left.+\mu \mathrm{e}^{\left(a_{i}-\alpha\right) t_{k}}\right\} \\
& \leq \mathrm{e}^{-\left(a_{i}-\alpha\right) t} \sum_{0<t_{k}<T_{i}}\left\{p_{i k}\left|y_{i}\left(t_{k}\right)\right| \mathrm{e}^{a_{i} t_{k}}\right\} \\
& +\mathrm{e}^{-\left(a_{i}-\alpha\right) t} p_{i} \varepsilon \int_{T_{i}}^{t} \mathrm{e}^{\left(a_{i}-\alpha\right) s} \mathrm{~d} s \\
& +\mathrm{e}^{-\left(a_{i}-\alpha\right) t} p_{i} \varepsilon \mu \mathrm{e}^{\left(a_{i}-\alpha\right) t} \\
& \leq \mathrm{e}^{-\left(a_{i}-\alpha\right) t} \sum_{0<t_{k}<T_{i}}\left\{p_{i k}\left|y_{i}\left(t_{k}\right)\right| \mathrm{e}^{a_{i} t_{k}}\right\} \\
& +\frac{p_{i} \varepsilon}{a_{i}-\alpha}+p_{i} \varepsilon \mu
\end{aligned}
$$

which means that $W_{5} \rightarrow 0$ as $t \rightarrow \infty$.

Now, we can derive from (27) that $\mathrm{e}^{\alpha t} \pi\left(y_{i}\right)(t) \rightarrow 0$ as $t \rightarrow \infty$ for $i \in \mathcal{N}$. It is therefore concluded that $\pi\left(y_{i}\right)(t) \subset$ $\mathscr{H}_{i}$ which results in $\pi(\mathscr{H}) \subset \mathscr{H}$.

Step 2. We need to prove that $\pi$ is contractive. For $\overline{\mathbf{z}}=$ $\left(z_{1}(t), \ldots, z_{n}(t)\right) \in \mathscr{H}$ and $\overline{\mathbf{y}}=\left(y_{1}(t), \ldots, y_{n}(t)\right) \in \mathscr{H}$, we estimate

$$
\left|\pi\left(y_{i}\right)(t)-\pi\left(z_{i}\right)(t)\right| \leq J_{1}+J_{2}+J_{3}+J_{4}
$$

where

$$
\begin{array}{r}
J_{1}=\mathrm{e}^{-a_{i} t} \int_{0}^{t} \mathrm{e}^{a_{i} s} \sum_{j=1}^{n}\left[\left|b_{i j}\right|\left|f_{j}\left(y_{j}(s)\right)-f_{j}\left(z_{j}(s)\right)\right|\right] \mathrm{d} s, \\
J_{2}=\mathrm{e}^{-a_{i} t} \int_{0}^{t} \mathrm{e}^{a_{i} s} \sum_{j=1}^{n}\left[\left|c_{i j}\right| \mid g_{j}\left(y_{j}\left(s-\tau_{i j}(s)\right)\right)\right. \\
\left.-g_{j}\left(z_{j}\left(s-\tau_{i j}(s)\right)\right) \mid\right] \mathrm{d} s
\end{array}
$$

$$
\begin{array}{r}
J_{3}=\mathrm{e}^{-a_{i} t} \int_{0}^{t} \mathrm{e}^{a_{i} s} \sum_{j=1}^{n}\left|d_{i j}\right| \int_{0}^{\rho(s)} \mid \sigma_{j}\left(y_{j}(s-\theta)\right) \\
-\sigma_{j}\left(z_{j}(s-\theta)\right) \mid \mathrm{d} \theta \mathrm{d} s \\
J_{4}=\mathrm{e}^{-a_{i} t} \sum_{0<t_{k}<t}\left\{\mathrm{e}^{a_{i} t_{k}}\left|I_{i k}\left(y_{i}\left(t_{k}\right)\right)-I_{i k}\left(z_{i}\left(t_{k}\right)\right)\right|\right\} .
\end{array}
$$

Note that

$$
\begin{aligned}
J_{1} \leq & \mathrm{e}^{-a_{i} t} \int_{0}^{t} \mathrm{e}^{a_{i} s} \sum_{j=1}^{n}\left[\left|b_{i j} l_{j}\right|\left|y_{j}(s)-z_{j}(s)\right|\right] \mathrm{d} s \\
\leq & \max _{j \in \mathcal{N}}\left|b_{i j} l_{j}\right| \sum_{j=1}^{n}\left\{\sup _{s \in[0, t]}\left|y_{j}(s)-z_{j}(s)\right|\right\} \\
& \times \mathrm{e}^{-a_{i} t} \int_{0}^{t} \mathrm{e}^{a_{i} s} \mathrm{~d} s \\
\leq & \frac{1}{a_{i}} \max _{j \in \mathcal{N}}\left|b_{i j} l_{j}\right| \sum_{j=1}^{n}\left\{\sup _{s \in[0, t]}\left|y_{j}(s)-z_{j}(s)\right|\right\}, \\
J_{2} \leq & \mathrm{e}^{-a_{i} t} \int_{0}^{t} \mathrm{e}^{a_{i} s} \sum_{j=1}^{n}\left[\left|c_{i j} k_{j}\right| \mid y_{j}\left(s-\tau_{i j}(s)\right)\right. \\
\leq & \max _{j \in \mathcal{N}}\left|c_{i j} k_{j}\right| \sum_{j=1}^{n}\left\{\sup _{\xi \in[-\tau, t]}\left|y_{j}(\xi)-z_{j}(\xi)\right|\right\} \\
\leq & \frac{1}{a_{i}} \max _{j \in \mathcal{N}}\left|c_{i j} k_{j}\right| \sum_{j=1}^{n}\left\{\sup _{\xi \in[-\tau, t]}\left|y_{j}(\xi)-z_{j}(\xi)\right|\right\}, \\
& \times \mathrm{e}^{-a_{i} t} \int_{0}^{t} \mathrm{e}^{a_{i} s} \mathrm{~d} s
\end{aligned}
$$$$
J_{3} \leq \mathrm{e}^{-a_{i} t} \int_{0}^{t} \mathrm{e}^{a_{i} s} \sum_{j=1}^{n}\left\{\left|d_{i j}\right| \int_{0}^{\rho(s)} \omega_{j} \mid y_{j}(s-\theta)\right.
$$$$
\left.-z_{j}(s-\theta) \mid \mathrm{d} \theta\right\} \mathrm{d} s
$$$$
\leq \mathrm{e}^{-a_{i} t} \int_{0}^{t} \mathrm{e}^{a_{i} s} \sum_{j=1}^{n}\left\{\left|d_{i j}\right| \sup _{\xi \in[s-\rho, s]}\left|y_{j}(\xi)-z_{j}(\xi)\right|\right.
$$$$
\left.\times \int_{0}^{\rho(s)} \omega_{j} \mathrm{~d} \theta\right\} \mathrm{d} s
$$$$
\leq \mathrm{e}^{-a_{i} t} \int_{0}^{t} \mathrm{e}^{a_{i} s} \sum_{j=1}^{n}\left\{\omega_{j}\left|d_{i j}\right| \sup _{\xi \in[-\rho, t]} \mid y_{j}(\xi)\right.
$$

$\left.-z_{j}(\xi) \mid \rho(s)\right\} \mathrm{d} s$ 


$$
\begin{aligned}
\leq & \max _{j \in \mathcal{N}}\left\{\omega_{j}\left|d_{i j}\right|\right\} \sum_{j=1}^{n}\left\{\sup _{\xi \in[-\rho, t]}\left|y_{j}(\xi)-z_{j}(\xi)\right|\right\} \\
& \times \mathrm{e}^{-a_{i} t} \int_{0}^{t} \mathrm{e}^{a_{i} s} \rho(s) \mathrm{d} s \\
\leq & \frac{\rho}{a_{i}} \max _{j \in \mathcal{N}}\left\{\omega_{j}\left|d_{i j}\right|\right\} \sum_{j=1}^{n}\left\{\sup _{\xi \in[-\rho, t]}\left|y_{j}(\xi)-z_{j}(\xi)\right|\right\} \\
J_{4} \leq & \mathrm{e}^{-a_{i} t} \sum_{0<t_{k}<t}\left\{\mathrm{e}^{a_{i} t_{k}} p_{i k}\left|y_{i}\left(t_{k}\right)-z_{i}\left(t_{k}\right)\right|\right\} \\
\leq & p_{i} \mathrm{e}^{-a_{i} t} \sup _{s \in[0, t]}\left|y_{i}(s)-z_{i}(s)\right| \sum_{0<t_{k}<t}\left\{\mathrm{e}^{a_{i} t_{k}} \mu\right\} \\
\leq & p_{i} \mathrm{e}^{-a_{i} t} \sup _{s \in[0, t]}\left|y_{i}(s)-z_{i}(s)\right| \\
\leq & p_{i}\left(\mu+\frac{1}{a_{i}}\right) \sup _{s \in[0, t]}\left|y_{i}(s)-z_{i}(s)\right| . \\
\leq & p_{i} \sup _{s \in[0, t]}\left|y_{i}(s)-z_{i}(s)\right| \mathrm{e}^{-a_{i} t}\left\{\int_{0<t_{r}<t_{k}}\left\{\mathrm{e}^{a_{i} s} \mathrm{~d} \mathrm{~s}+\mathrm{e}^{a_{i} t} \mu\right\}\right. \\
& \left.\left\{\mathrm{a}_{a_{i} t_{r}}\left(t_{r+1}-t_{r}\right)\right\}+\mathrm{e}^{a_{i} t_{k}} \mu\right\}
\end{aligned}
$$

It is then derived from (36) that

$$
\begin{aligned}
& \sup _{t \in\left[-m^{*}, T\right]}\left|\pi\left(y_{i}\right)(t)-\pi\left(z_{i}\right)(t)\right| \\
& \leq \frac{1}{a_{i}} \max _{j \in \mathcal{N}}\left|b_{i j} l_{j}\right| \sum_{j=1}^{n}\left\{\sup _{s \in\left[-m^{*}, T\right]}\left|y_{j}(s)-z_{j}(s)\right|\right\} \\
& \quad+\frac{1}{a_{i}} \max _{j \in \mathcal{N}}\left|c_{i j} k_{j}\right| \sum_{j=1}^{n}\left\{\sup _{\xi \in\left[-m^{*}, T\right]}\left|y_{j}(\xi)-z_{j}(\xi)\right|\right\} \\
& +\frac{\rho}{a_{i}} \max _{j \in \mathcal{N}}\left\{\omega_{j}\left|d_{i j}\right|\right\} \sum_{j=1}^{n}\left\{\sup _{\xi \in\left[-m^{*}, T\right]}\left|y_{j}(\xi)-z_{j}(\xi)\right|\right\} \\
& \quad+p_{i}\left(\mu+\frac{1}{a_{i}}\right) \sup _{s \in\left[-m^{*}, T\right]}\left|y_{i}(s)-z_{i}(s)\right|,
\end{aligned}
$$

which means that

$$
\begin{aligned}
& \sum_{i=1}^{n} \sup _{t \in\left[-m^{*}, T\right]}\left|\pi\left(y_{i}\right)(t)-\pi\left(z_{i}\right)(t)\right| \\
& \quad \leq \chi \sum_{j=1}^{n}\left\{\sup _{s \in\left[-m^{*}, T\right]}\left|y_{j}(s)-z_{j}(s)\right|\right\},
\end{aligned}
$$

where

$$
\begin{aligned}
\chi \triangleq & \sum_{i=1}^{n}\left\{\frac{1}{a_{i}} \max _{j \in \mathcal{N}}\left|b_{i j} l_{j}\right|+\frac{1}{a_{i}} \max _{j \in \mathcal{N}}\left|c_{i j} k_{j}\right|+\frac{\rho}{a_{i}} \max _{j \in \mathcal{N}}\left|\omega_{j} d_{i j}\right|\right\} \\
& +\max _{i \in \mathcal{N}}\left\{p_{i}\left(\mu+\frac{1}{a_{i}}\right)\right\} .
\end{aligned}
$$

In view of condition (iii), we know that $\pi$ is a contraction mapping, and hence, there exists a unique fixed point $\overline{\mathbf{y}}(\cdot)$ of $\pi$ in $\mathscr{H}$ which means that $\overline{\mathbf{y}}^{\mathrm{T}}(\cdot)$ is the solution to (1)-(3) and $\mathrm{e}^{\alpha t}\left\|\overline{\mathbf{y}}^{\mathrm{T}}(\cdot)\right\| \rightarrow 0$ as $t \rightarrow \infty$. This completes the proof.

Lemma 4. Assume conditions (A1)-(A4) hold. Provided that

(i) $\inf _{k=1,2, \ldots}\left\{t_{k}-t_{k-1}\right\} \geq 1$,

(ii) there exist constants $p_{i}$ such that $p_{i k} \leq p_{i}$ for $i \in \mathcal{N}$ and $k=1,2, \ldots$,

(iii) $\sum_{i=1}^{n}\left\{\left(1 / a_{i}\right) \max _{j \in \mathcal{N}}\left|b_{i j} l_{j}\right|+\left(1 / a_{i}\right) \max _{j \in \mathcal{N}}\left|c_{i j} k_{j}\right|+\right.$ $\left.\left(\rho / a_{i}\right) \max _{j \in \mathcal{N}}\left|\omega_{j} d_{i j}\right|\right\}+\max _{i \in \mathcal{N}}\left\{p_{i}\left(1+\left(1 / a_{i}\right)\right)\right\} \triangleq \chi<$ 1 ,

then the trivial equilibrium $\mathbf{x}=0$ is globally exponentially stable.

Proof. Lemma 4 is a direct conclusion by letting $\mu=1$ in Theorem 3.

Remark 5. In Theorem 3, we use the fixed point theorem to prove the existence and uniqueness of solution and the global exponential stability of trivial equilibrium all at once, while Lyapunov method fails to do this.

Remark 6. The presented sufficient conditions in Theorem 3 and Lemma 4 do not require even the differentiability of delays, let alone the monotone decreasing behavior of delays which is necessary in some relevant works.

\section{Example}

Consider the following two-dimensional impulsive cellular neural network with time-varying and distributed delays.

$$
\begin{aligned}
& \frac{\mathrm{d} x_{i}(t)}{\mathrm{d} t}=-a_{i} x_{i}(t)+\sum_{j=1}^{2} b_{i j} f_{j}\left(x_{j}(t)\right) \\
&+\sum_{j=1}^{2} c_{i j} g_{j}\left(x_{j}\left(t-\tau_{i j}(t)\right)\right) \\
&+\sum_{j=1}^{2} d_{i j} \int_{0}^{\rho(t)} \sigma_{j}\left(x_{j}(t-\theta)\right) \mathrm{d} \theta, \quad t \geq 0, \quad t \neq t_{k}, \\
& \Delta x_{i}\left(t_{k}\right)= x_{i}\left(t_{k}+0\right)-x_{i}\left(t_{k}\right)=\arctan \left(0.4 x_{i}\left(t_{k}\right)\right), \\
& k=1,2, \ldots,
\end{aligned}
$$


with the initial conditions $x_{1}(s)=\cos (s), x_{2}(s)=\sin (s)$ on $-m^{*} \leq s \leq 0$, where $\tau_{i j}(t)=0.8+0.4 \cos (t), \rho(t)=0.5+$ $0.3 \sin (t), m^{*}$ is defined as shown in (3), $a_{1}=a_{2}=7, b_{i j}=$ $0, c_{11}=0, c_{12}=1 / 7, c_{21}=-1 / 7, c_{22}=-1 / 7, d_{11}=3 / 7$, $d_{12}=2 / 7, d_{21}=0, d_{22}=1 / 7, f_{j}(s)=g_{j}(s)=\sigma_{j}(s)=$ $(|s+1|-|s-1|) / 2$, and $t_{k}=t_{k-1}+0.5 k$.

It is easily to find that $\mu=0.5, l_{j}=k_{j}=\omega_{j}=1$, and $p_{i k}=0.4$. Let $p_{i}=0.8$ and compute

$$
\begin{aligned}
\sum_{i=1}^{2}\{ & \left.\frac{1}{a_{i}} \max _{j \in \mathcal{N}}\left|b_{i j} l_{j}\right|+\frac{1}{a_{i}} \max _{j \in \mathcal{N}}\left|c_{i j} k_{j}\right|+\frac{\rho}{a_{i}} \max _{j \in \mathcal{N}}\left|\omega_{j} d_{i j}\right|\right\} \\
& +\max _{i \in \mathcal{N}}\left\{p_{i}\left(\mu+\frac{1}{a_{i}}\right)\right\}<1 .
\end{aligned}
$$

From Theorem 3, we conclude that the trivial equilibrium $\mathbf{x}=0$ of this two-dimensional impulsive cellular neural network with time-varying and distributed delays is globally exponentially stable.

\section{Conclusions}

This article is a new attempt of applying the fixed point theory to the stability analysis of impulsive neural networks with time-varying and distributed delays, which is different from the existing relevant publications where Lyapunov theory is the main technique. From what have been discussed above, we see that the contraction mapping principle is effective for not only the investigation of the existence and uniqueness of solution but also for the stability analysis of trivial equilibrium. In the future, we will continue to explore the application of other kinds of fixed point theorems to the stability research of complex neural networks.

\section{Acknowledgment}

This work is supported by the National Natural Science Foundation of China under Grant no. 61174077 and Natural Science Foundation of Jiangsu of China under Grant no. BK2012682.

\section{References}

[1] L. O. Chua and L. Yang, "Cellular neural networks: theory," IEEE Transactions on Circuits and Systems, vol. 35, no. 10, pp. 1257$1272,1988$.

[2] L. O. Chua and L. Yang, "Cellular neural networks: applications," IEEE Transactions on Circuits and Systems, vol. 35, no. 10, pp. 1273-1290, 1988.

[3] G. T. Stamov and I. M. Stamova, "Almost periodic solutions for impulsive neural networks with delay," Applied Mathematical Modelling, vol. 31, no. 7, pp. 1263-1270, 2007.

[4] S. Ahmad and I. M. Stamova, "Global exponential stability for impulsive cellular neural networks with time-varying delays," Nonlinear Analysis. Theory, Methods \& Applications, vol. 69, no. 3, pp. 786-795, 2008.

[5] K. Li, X. Zhang, and Z. Li, "Global exponential stability of impulsive cellular neural networks with time-varying and distributed delay," Chaos, Solitons and Fractals, vol. 41, no. 3, pp. 1427-1434, 2009.
[6] J. Qiu, "Exponential stability of impulsive neural networks with time-varying delays and reaction-diffusion terms," Neurocomputing, vol. 70, no. 4-6, pp. 1102-1108, 2007.

[7] X. Wang and D. Xu, "Global exponential stability of impulsive fuzzy cellular neural networks with mixed delays and reactiondiffusion terms," Chaos, Solitons \& Fractals, vol. 42, no. 5, pp. 2713-2721, 2009.

[8] Y. Zhang and Q. Luo, "Global exponential stability of impulsive delayed reaction-diffusion neural networks via Hardy-Poincare Inequality," Neurocomputing, vol. 83, pp. 198-204, 2012.

[9] Y. Zhang and Q. Luo, "Novel stability criteria for impulsive delayed reaction-diffusion Cohen-Grossberg neural networks via Hardy-Poincarè inequality," Chaos, Solitons \& Fractals, vol. 45, no. 8, pp. 1033-1040, 2012.

[10] W. Zhang, Y. Tang, J.-a. Fang, and X. Wu, "Stability of delayed neural networks with time-varying impulses," Neural Networks, vol. 36, pp. 59-63, 2012.

[11] S. Zhu and Y. Shen, "Robustness analysis for connection weight matrices of global exponential stability of stochastic recurrent neural networks," Neural Networks, vol. 38, pp. 17-22, 2013.

[12] T. A. Burton, Stability by Fixed Point Theory for Functional Differential Equations, Dover, Mineola, NY, USA, 2006.

[13] L. C. Becker and T. A. Burton, "Stability, fixed points and inverses of delays," Proceedings of the Royal Society of Edinburgh A, vol. 136, no. 2, pp. 245-275, 2006.

[14] T. A. Burton, "Fixed points, stability, and exact linearization," Nonlinear Analysis. Theory, Methods \& Applications, vol. 61, no. 5, pp. 857-870, 2005.

[15] T. A. Burton, "Fixed points, Volterra equations, and Becker's resolvent," Acta Mathematica Hungarica, vol. 108, no. 3, pp. 261281, 2005.

[16] T. A. Burton, "Fixed points and stability of a nonconvolution equation," Proceedings of the American Mathematical Society, vol. 132, no. 12, pp. 3679-3687, 2004.

[17] T. A. Burton, "Perron-type stability theorems for neutral equations," Nonlinear Analysis. Theory, Methods \& Applications, vol. 55, no. 3, pp. 285-297, 2003.

[18] T. A. Burton, "Integral equations, implicit functions, and fixed points," Proceedings of the American Mathematical Society, vol. 124, no. 8, pp. 2383-2390, 1996.

[19] T. A. Burton and T. Furumochi, "Krasnoselskii's fixed point theorem and stability," Nonlinear Analysis. Theory, Methods \& Applications, vol. 49, no. 4, pp. 445-454, 2002.

[20] T. A. Burton and B. Zhang, "Fixed points and stability of an integral equation: nonuniqueness," Applied Mathematics Letters, vol. 17, no. 7, pp. 839-846, 2004.

[21] T. Furumochi, "Stabilities in FDEs by Schauder's theorem," Nonlinear Analysis, Theory, Methods and Applications, vol. 63, no. 5-7, pp. e217-e224, 2005.

[22] C. Jin and J. Luo, "Fixed points and stability in neutral differential equations with variable delays," Proceedings of the American Mathematical Society, vol. 136, no. 3, pp. 909-918, 2008.

[23] Y. N. Raffoul, "Stability in neutral nonlinear differential equations with functional delays using fixed-point theory," Mathematical and Computer Modelling, vol. 40, no. 7-8, pp. 691-700, 2004.

[24] B. Zhang, "Fixed points and stability in differential equations with variable delays," Nonlinear Analysis, Theory, Methods and Applications, vol. 63, no. 5-7, pp. e233-e242, 2005.

[25] J. Luo, "Fixed points and stability of neutral stochastic delay differential equations," Journal of Mathematical Analysis and Applications, vol. 334, no. 1, pp. 431-440, 2007. 
[26] J. Luo, "Fixed points and exponential stability of mild solutions of stochastic partial differential equations with delays," Journal of Mathematical Analysis and Applications, vol. 342, no. 2, pp. 753-760, 2008.

[27] J. Luo, "Stability of stochastic partial differential equations with infinite delays," Journal of Computational and Applied Mathematics, vol. 222, no. 2, pp. 364-371, 2008.

[28] J. Luo and T. Taniguchi, "Fixed points and stability of stochastic neutral partial differential equations with infinite delays," Stochastic Analysis and Applications, vol. 27, no. 6, pp. 1163-1173, 2009.

[29] R. Sakthivel and J. Luo, "Asymptotic stability of impulsive stochastic partial differential equations with infinite delays," Journal of Mathematical Analysis and Applications, vol. 356, no. 1, pp. 1-6, 2009.

[30] R. Sakthivel and J. Luo, "Asymptotic stability of nonlinear impulsive stochastic differential equations," Statistics \& Probability Letters, vol. 79, no. 9, pp. 1219-1223, 2009.

[31] J. Luo, "Fixed points and exponential stability for stochastic Volterra-Levin equations," Journal of Computational and Applied Mathematics, vol. 234, no. 3, pp. 934-940, 2010.

[32] D. R. Smart, Fixed Point Theorems, Cambridge University Press, London, UK, 1974. 


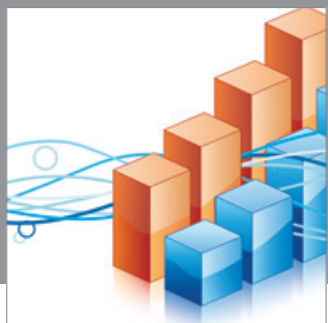

Advances in

Operations Research

mansans

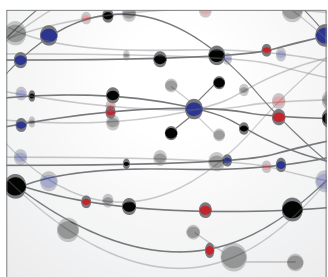

The Scientific World Journal
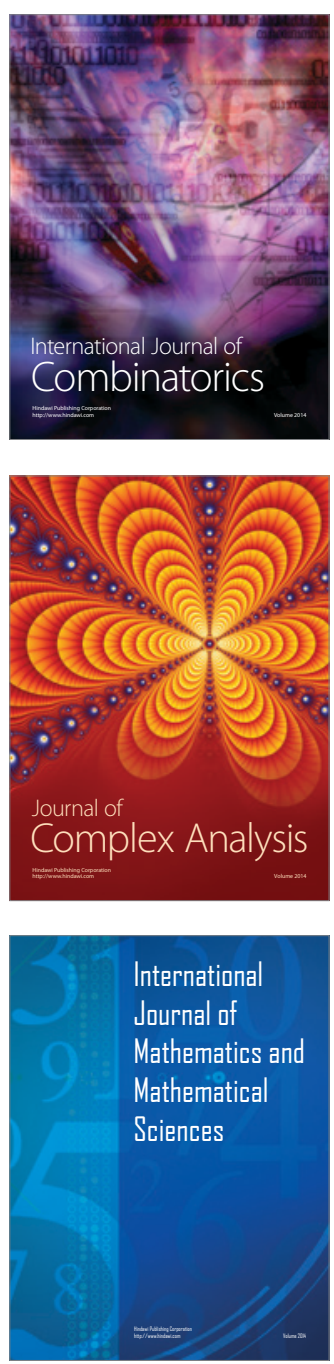
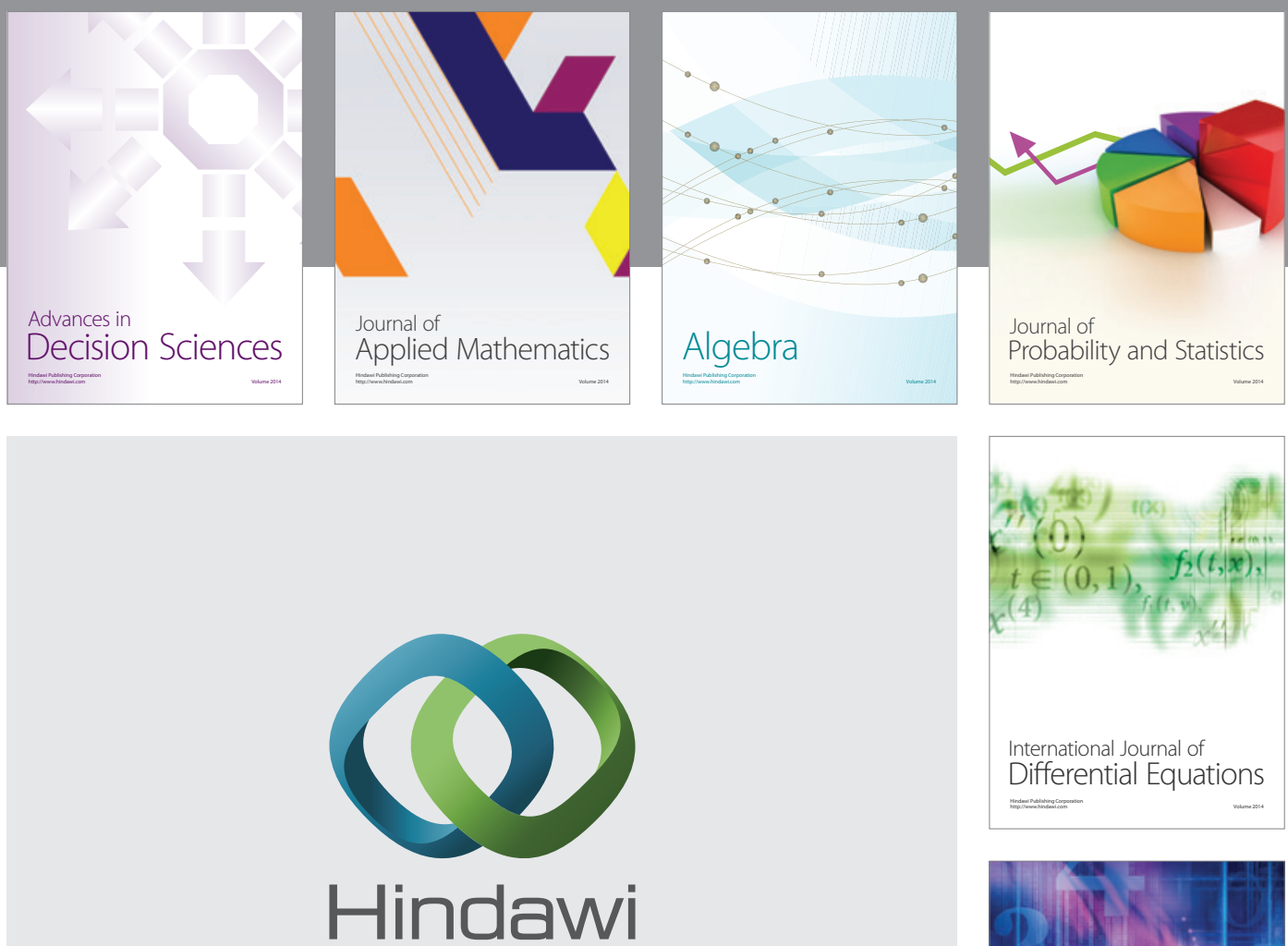

Submit your manuscripts at http://www.hindawi.com
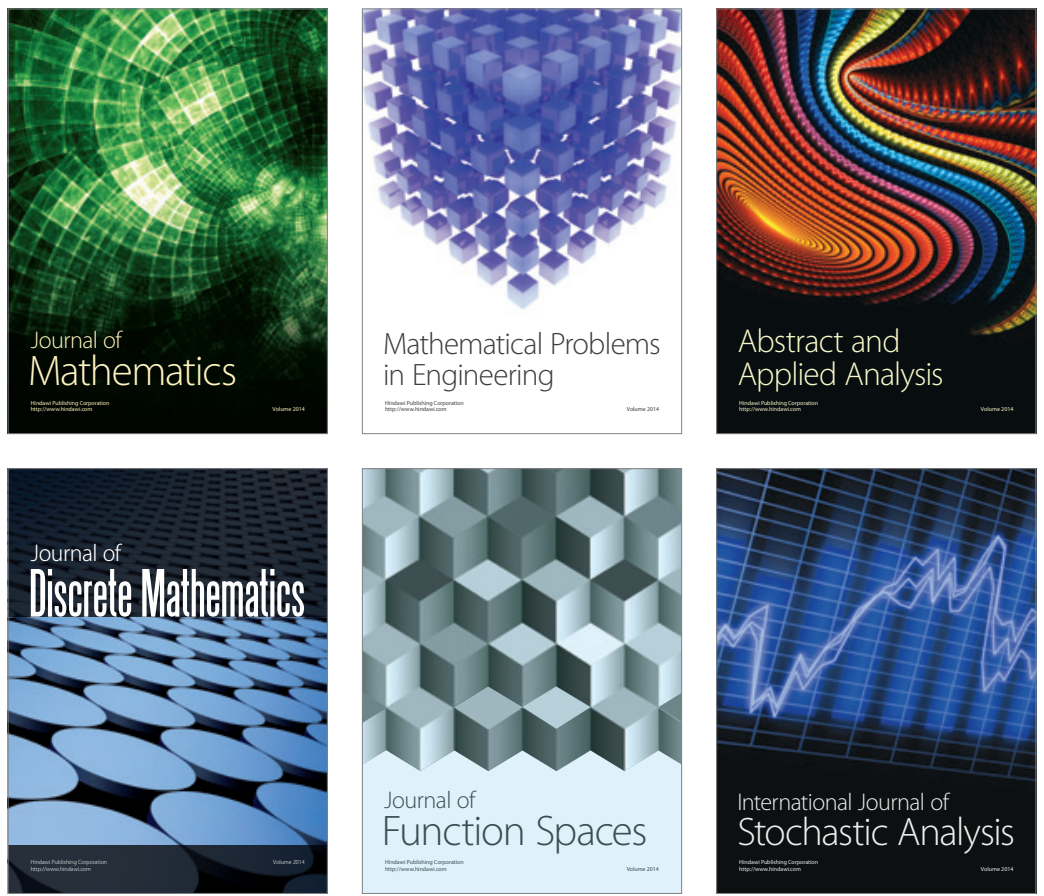

Journal of

Function Spaces

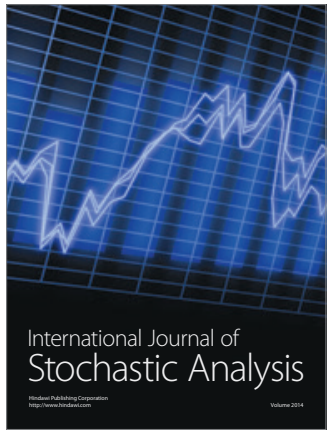

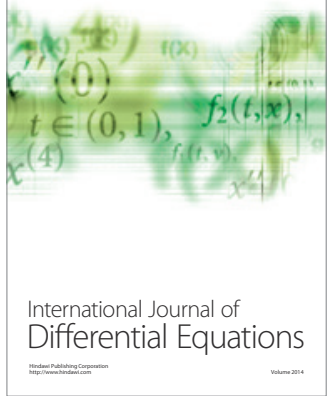
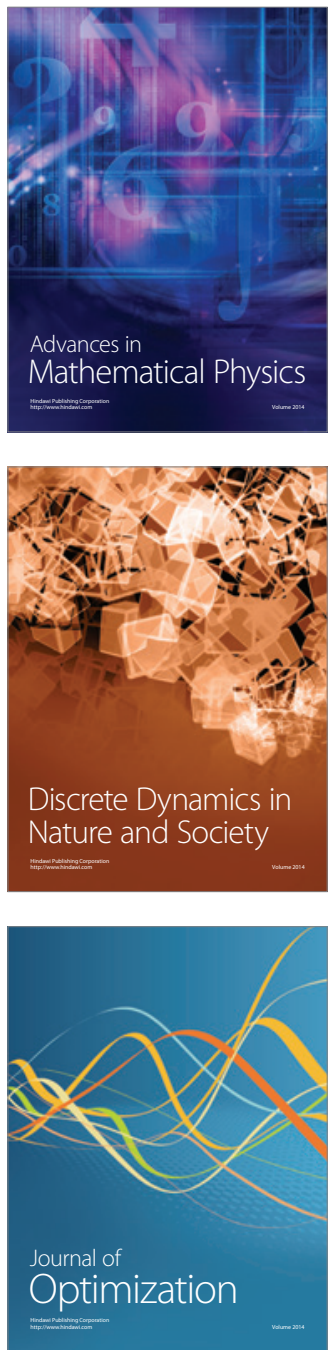